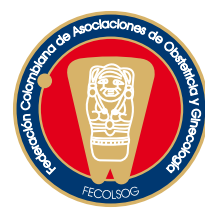

\title{
Editorial
}

\section{ESTAMOS EN DEUDA CON LOS DERECHOS SEXUALES Y REPRODUCTIVOS EN AMÉRICA LATINA Y EL CARIBE}

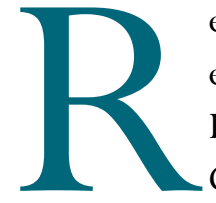
ecibí la invitación de escribir el editorial para este número de la Revista Colombiana de Obstetricia y Ginecología y no dudé en aceptarla, no solo por el honor que ello representa para mí, sino además por la responsabilidad de reiterar la defensa de los Derechos Sexuales y Reproductivos (DSR) de las mujeres de América Latina y El Caribe que la Federación Latinoamericana de Sociedades de Obstetricia y Ginecología (FLASOG) ha implementado desde el año 2002.

En el escenario del Congreso Latinoamericano de Obstetricia y Ginecología, la Asamblea de FLASOG aprobó la Declaración de Santa Cruz que asumió la defensa de los siguientes DSR:

- Maternidad saludable y segura, sin riesgo de morir.

- Vida sexual libre de violencia, del riesgo de contraer una infección de transmisión sexual y un embarazo no deseado (END).

- Regulación de la fecundidad mediante el acceso a los métodos anticonceptivos que incluyen la anticoncepción de emergencia.

- Interrupción del embarazo dentro del marco de la ley.

- Información y derecho al acceso de los servicios de salud sexual reproductiva (SSR).

El cuidado de la salud debe enfocarse en los Derechos

Humanos (DDHH) que se fundamentan en el respeto hacia la dignidad de las personas. Implica igualdad de trato y no discriminación en la atención de la salud. ${ }^{1}$ Los DSR de las personas, y en particular de las mujeres son parte inalienable, integral e indivisible de los DDHH que son universales, interdependientes e interrelacionados. ${ }^{2}$
Sin embargo, aún existen grandes preocupaciones y deficiencias en materia de SSR, como la escasez de recursos, la violación de los DDHH de las mujeres, incluyendo los DSR, por lo cual la salud sexual reproductiva resulta comprometida, no por falta de conocimientos médicos, sino a causa de las violaciones de los DDHH de las mujeres. ${ }^{3}$ Según las estadísticas, seis de cada diez mujeres sufren violencia física o sexual a lo largo de su vida, del 7 al 36\% padecen violencia sexual (VS) en la niñez y del 6 al 59\% sufren VS después de los 15 años, y es la pareja íntima la más involucrada en estos actos. ${ }^{4}$ Las graves repercusiones que tiene la violencia sobre las mujeres están muy bien identificadas, puesto que pueden ocasionar consecuencias a corto y a largo plazo en la salud de las mujeres, incluyendo el trauma físico, la infección por VIH, el END y el aborto inseguro. El trauma psicológico resultante puede tener un efecto negativo sobre la conducta y relaciones sexuales, la capacidad para negociar el sexo seguro y un potencial aumento en el abuso de drogas. ${ }^{5}$ Es clamorosa en América Latina la falta de servicios que atiendan este problema, que además del abordaje temprano de la VS, realicen una atención integral que mantenga:

- Enlaces médico-jurídicos que funcionen para permitir el cumplimiento de la justicia y la prestación de servicios de salud.

- Entrega de servicios posviolación.

- Recolección y entrega de evidencias al sistema judicial.

- Acceso a las referencias, con protocolos de tratamiento y procedimientos médico-jurídicos estandarizados. ${ }^{6,7}$

Para poder permitir el derecho de las mujeres a vivir una vida sexual libre de violencia, todavía 
está pendiente en nuestros países: empoderar a las mujeres, implementar y/o ampliar y mejorar los servicios de SSR, detectar en la rutina de la atención diaria los casos de violencia contra las mujeres, hacer una atención temprana e integral de la VS y coordinar con otros servicios de salud y jurídico-legales para no revictimizarlas.

El gran reto que enfrentamos es el poco interés que aún existe frente a la mortalidad materna (MM) entre quienes toman las decisiones. La MM es el indicador más sensible del nivel de atención de la SSR, porque usualmente expresa las grandes brechas existentes dentro de las poblaciones, en donde son las mujeres más desprotegidas, excluidas y discriminadas las que terminan su embarazo con una trágica muerte. ${ }^{8}$ Sabemos que las intervenciones más eficaces para enfrentar las muertes son: el cuidado intraparto en las unidades de salud por personal calificado, cuidados obstétricos de emergencia, cuidado prenatal institucional, la reducción de las brechas de acceso a los servicios, la atención del parto con enfoque intercultural, el cuidado posparto, planificación familiar y el aborto en condiciones seguras. ${ }^{9}$

Respecto a estos dos últimos aspectos vale la pena registrar que la planificación familiar $(\mathrm{PF})$ y la anticoncepción, gracias a las tecnologías disponibles hoy en día, juegan un rol importante para reducir la $\mathrm{MM}$, al restringir el número de END, disminuir la tasa de fecundidad y por tanto reducir el riesgo que representan el embarazo y el parto. ${ }^{10}$ Se puede afirmar que de 1/4 a 2/5 partes de la MM puede disminuir si se evitan los END. ${ }^{11}$ En América Latina se han hecho avances, pero aún existen grupos poblacionales en los que ocurre una importante demanda insatisfecha de métodos de planificación familiar. ${ }^{12}$ Se requiere en este sentido ampliar los servicios de PF, disponer de una gama mayor de métodos anticonceptivos, incluyendo la anticoncepcion de emergencia, asegurar una buena logística y mejorar la calidad de atención. ${ }^{13}$

Por otra parte, a pesar de la alta prevalencia de de diversos métodos anticonceptivos y de métodos seguros y eficaces de aborto, al 2008 ocurren en el mundo 42 millones de abortos inducidos, de los cuales
21.6 millones son abortos inseguros. En América Latina hay más de 4 millones de abortos inseguros, constituyendo la frecuencia más alta del mundo (31 por cada 1000 mujeres en edad fértil) que aún ocasionan cifras importantes de MM, a pesar de que la morbilidad por aborto ha disminuido, probablemente por el mayor acceso y uso del misoprostol. El aborto es más frecuente en los países con menor prevalencia de anticonceptivos seguros y con legislación restrictiva, como es el caso de nuestra América. ${ }^{14-16}$

Una reciente publicación de la Organización Mundial de la Salud (OMS), ${ }^{17}$ muestra importantes avances en la reducción de la razón de muerte materna, en la mayoría de regiones, excepto en el África. Sin embargo, esto no ha sido suficiente para poder alcanzar la meta del milenio de reducir en $3 / 4$ partes la MM. Para obtener este fin, será necesario enfatizar intervenciones complementarias como:

- Movilización de los comités de MM.

- Mejorar los registros.

- Utilizar la Clasificación Internacional de Enfermedades (CIE).

- Empoderar a las mujeres mediante una mejor información y acceso a la educación.

- Igualdad de oportunidades.

- El reconocimiento del derecho a tomar sus propias decisiones.

- Ampliar y mejorar la calidad de los servicios.

- Atención humanizada del embarazo y parto con criterio integral de salud.

- Cuidar de los embarazos que ponen en riesgo la salud y la vida de las mujeres, como también de los END, frente a lo cual, el Population Reference Bureau sostiene que todo embarazo debe ser deseado, que la fecundidad debe ser lograda por elección.

La Federación Internacional de Ginecología y Obstetricia (FIGO), ha identificado este grave problema de salud y ha puesto en marcha la Iniciativa Prevención del Aborto Inseguro (descrita para Colombia en este número por el Dr. Pío Iván Gómez) que se viene operando en 43 países del mundo, de los cuales 16 corresponden a Latinoamérica y el Caribe. El objetivo 
es reducir el número de abortos y en consecuencia las muertes maternas ${ }^{18}$, con una intervención integral que logre prevenir los END por medio de la PF y la educación sexual, posibilitando el acceso de las mujeres al aborto seguro en los términos que establece la ley de cada país, para poder ofrecer una adecuada atención posaborto, rápida, de preferencia ambulatoria y con entrega de anticonceptivos posaborto. ${ }^{19}$

Los avances en este tema, se han logrado utilizando los factores facilitadores e identificando barreras, pero no es suficiente con identificarlas para poder alcanzar las metas propuestas del milenio en el año 2015. Es necesario asumir las siguientes acciones: el compromiso de quienes toman decisiones y de los profesionales de la salud, estimular la mayor provisión de recursos, mejorar los registros, utilizar tecnologías adecuadas, mejorar el cumplimiento de la legislación vigente y cuando sea necesario revisarla. Todo lo anterior, con el propósito de evitar muertes y sufrimientos innecesarios, al acercarnos con mayor respeto a los DDHH y como consecuencia al respeto por los DSR.

\section{Dr. Luis Távara Orozco}

Coordinador del Comité de Derechos Sexuales y Reproductivos, FLASOG. Coordinador Regional para los países de América del Sur de la Iniciativa FIGO "Prevención del Aborto Inseguro".

\section{REFERENCIAS}

1. Fathalla MF. From Obstetrics and Gynecology to women's health: the road ahead. New York, London: The Parthenon Publishing Group; 1997.

2. UNFPA. Conferencia Internacional sobre la Población y Desarrollo. Cairo; 1994.

3. Cook RJ, Dickens BM, Fathalla MF. Salud Reproductiva y Derechos Humanos, 2a edición, traducida al español. Bogotá, Colombia: Profamilia; 2005. p. 605.

4. WHO. Violence against women. Geneva: WHO; June 2000.

5. Ellsberg M, Peña R, Herrera A, Wiskuist A, Hullgren G. Confites en el infierno. Prevalencia y características de la violencia conyugal hacia las mujeres en Nicaragua. Nicaragua: Red de mujeres contra la violencia/ Departamento de Medicina Preventiva y Salud Pública de la Facultad de Medicina UNAN-León/Departamento de Epidemiología y Salud Pública de la Universidad Umea, Sweden; 1998.

6. WHO. Guidelines for medico-legal care of victims of sexual violence. Geneva: WHO; 2003.

7. Ortiz JD, Rosas C, Távara L. Propuestas de estándares regionales para la elaboración de protocolos de atención integral temprana a víctimas de violencia sexual. Lima, Perú: Comité de Derechos Sexuales y Reproductivos de FLASOG, 2011. p. 88.

8. WHO. Reproductive health indicators for global monitoring. Geneva: WHO; 2001.

9. Campbell OM, Graham WJ; Lancet Maternal Survival Series steering group. Strategies to reduce maternal mortality: getting on with what works. Lancet 2006;368:1284-99

10. Population Reference Bureau. World population data sheet: demographic data and estimates for the countries and regions of the world. Washington DC: PRB; 2000.

11. Trussell J, Pebley AR. The potential impact of changes infertility on infant, child and maternal mortality. Stud Fam Plann 1984;15:267-80.

12. Singh S, Darroch JE, Ashford LS, Vlassoff M. Adding It Up: The benefits of investing in sexual and reproductive health Care. New York: Guttmacher Institute/UNFPA; 2003

13. Távara-Orozco L. Análisis de la oferta de anticonceptivos en el Perú. Rev Per Ginecol Obstet 2010;56:120-36.

14. Shah I, Ahman E. Unsafe abortion in 2008: global and regional levels and trends. Reproductive Health Matters 2010;18:90-101.

15. WHO. Unsafe Abortion. Global and regional estimates of the incidence of unsafe abortion and associated mortality in 2008. Geneva: WHO; 2010.

16. Harper CC, Blanchard K, Grossman D Henderson JT, Darney PD. Reducing maternal mortality due to elective abortion: potential impact of misoprostol in lowresource setting. Int J Gynecol Obstet 2007;98:66-9.

17. WHO, UNICEF, UNFPA, WORLD BANK. Trends in maternal mortality: 1990 to 2008.

18. Leke RJ, de Gil MP, Távara L, Faundes A. The FIGO working group on the prevention of unsafe abortion: mandate and process for achievement. Int J Gynecol Obstet 2010;110:S20-4.

19. Mirembe F, Karanja J, Hassan EO, Fáundes A. Goals and activities proposed by countries in seven regions of the world toward prevention of unsafe abortion. Int J Gynecol Obstet 2010;110:S25-9.

20. Fáundes A, Zaidi S. Prevention of unsafe abortion: analysis of the current situation and the task ahead. Int J Gynecol Obstet 2010;110:S38-42. 